\title{
Subwavelength-resolution label-free photoacoustic microscopy of optical absorption in vivo
}

\author{
Chi Zhang ${ }^{+}$, Konstantin Maslov ${ }^{+}$, and Lihong V. Wang ${ }^{*}$ \\ Optical Imaging Laboratory, Department of Biomedical Engineering, Washington University in St. \\ Louis, One Brookings Drive, St. Louis, MO 63130, USA
}

\begin{abstract}
Optical absorption provides essential biological functional information but cannot be sensed by mainstream optical microscopy technologies directly, which detect fluorescence or scattering and may require undesirable labeling. Here we developed in vivo subwavelength-resolution photoacoustic microscopy (SW-PAM) that provides exquisitely high optical-absorption contrast due to nonfluorescent, or fluorescent, endogenous pigments. Having approached the ultimate diffractionlimited optical resolution, SW-PAM can resolve subcellular organelles. Vasculature and early-stage melanoma were imaged with 12:1 and 17:1 contrasts, respectively, without labeling. SW-PAM along with the scaled-up macroscopy, as the only technology that measures the same contrast origin over such a wide length scale, can potentially accelerate translation from microscopic research to clinical practice.
\end{abstract}

Photoacoustic imaging [1,2] is an emerging biomedical imaging technology that utilizes the photoacoustic effect-absorbed light is converted to heat, subsequently inducing acoustic waves. If an optical objective is used to focus light into the biological tissue, and an ultrasonic transducer is focused on the same area to receive the photoacoustic signals, a high-resolution image of the tissue can be obtained. This approach, referred to as photoacoustic microscopy (PAM), has been successfully applied to in vivo structural, functional, and molecular imaging [3-6].

PAM differs from other forms of optical microscopy (OM) in contrast mechanisms. The confocal structure of PAM is similar to that of confocal optical microscopy, but PAM uses an ultrasonic transducer to detect the photoacoustic waves. In biological tissues, photons may be elastically scattered, inelastically scattered (converted to fluorescence or Raman signals), or absorbed [7]. Elastic-scattering OM—such as confocal microscopy or optical coherence tomography-detects the backscattered photons and therefore images scattering contrast. Fluorescence OM—such as confocal microscopy or two-photon microscopy—detects inelastically scattered photons from samples, normally labeled by exogenous fluorescent markers, so the contrast is sensitive to the concentration of fluorophores and the fluorescence quantum yield. However, most endogenous pigments have extremely weak fluorescence, where most absorbed energy is converted to heat. By contrast, PAM is sensitive to optical absorption with a relative sensitivity of $100 \%$ due to background-free detection [8].

PAM holds great potential for label-free imaging of melanoma and vasculature because nonfluorescent melanin and hemoglobin are major sources of endogenous absorption in

\footnotetext{
*Corresponding author: lhwang@biomed.wustl.edu.

+These authors contributed equally to this work.

OCIS codes: $170.3880,170.5120,180.5810$.
} 
biological tissue in the visible and near-infrared spectral range. Melanoma, arising from melanocytes, is the most deadly skin cancer [9]. The diagnosis of melanoma is based on inaccurate visual inspection and invasive biopsy. By providing in vivo, noninvasive, and highresolution imaging, PAM promises to diagnose melanoma in the early stage, which is the key to successful treatment. Moreover, as a hall mark of cancer, tumor angiogenesis is currently imaged either ex vivo by microscopic methods at high resolution or in vivo by clinical methods at low resolution [10]. With high endogenous contrast, PAM can identify angiogenic vessels in vivo.

In optical-resolution PAM, the numerical aperture (NA) of the optical objective is the keythe tighter the optical focus, the finer the image resolution. The first optical-resolution PAM system reached a resolution of $5 \mu \mathrm{m}$ [4]. Here, by using a water-immersion optical objective with a 1.23 NA, which is close to the ultimate limit, we have finally approached the highest diffraction-limited optical resolution and achieved subwavelength-resolution PAM (SWPAM)—220 nm resolution at $532 \mathrm{~nm}$ wavelength—as shown in Fig. 1.

In SW-PAM, a Nd:YVO4 laser generated pulses with a $532 \mathrm{~nm}$ wavelength $(\lambda)$ and a $1.5 \mathrm{~ns}$ duration. The pulses were coupled to a single-mode optical fiber, which was connected to the optical objective with a 1.23 NA. The sample was irradiated by the laser pulse focused by the objective, and the ultrasonic transducer (with a central frequency of $40 \mathrm{MHz}$ and a numerical aperture of 0.5 ) detected the resulting time-resolved photoacoustic wave in transmission mode. The typical pulse energy was $10 \mathrm{~nJ}$ for slide samples and $60 \mathrm{~nJ}$ for mouse ears. The signals were then amplified and digitized at a sampling rate of $1 \mathrm{GHz}$. The objective and the transducer mechanically scanned in raster mode in the $x-y$ plane with a scanning speed of $2 \mathrm{~mm} / \mathrm{s}$ and a step size of $125 \mathrm{~nm}$, which was controlled by a separate computer. After scanning, a maximumamplitude projection (MAP) image was obtained by projecting the maximum amplitude of each time-resolved signal onto the $x-y$ scanning plane.

In order to measure the transverse spatial resolution of SW-PAM, gold nanospheres with a diameter of $15 \mathrm{~nm}$ were imaged. A typical sphere was chosen, and the averaged pixel value was calculated with respect to the distance from the sphere center [Fig. 1(c)]. Then the data was fitted by the theoretical Bessel-form function. The system resolution, given by the full width at half maximum (FWHM) of the Bessel-form point spread function, is $220 \pm 20 \mathrm{~nm}$, agreeing well with the theoretical value $0.51 \lambda / \mathrm{NA} \approx 221 \mathrm{~nm}$.

To validate SW-PAM with wide-field OM, we imaged ex vivo melanoma cells and red blood cells. The PAM images have a dark background while the OM images have a bright background. The bright (white) dots in the PAM image of melanoma cells are melanosomes -organelles containing melanin [Fig. 2(a), left]. However, the melanosomes appear dark in the OM image [Fig. 2(a), middle] because their absorption attenuated the light transmission. The contrast between melanosomes and other areas in the PAM image [(54.5 \pm 0.4$): 1]$ is much higher than that in the OM image $[(0.79 \pm 0.04): 1]$ because PAM is sensitive to only absorption, but $\mathrm{OM}$ shows both absorption and scattering (the latter is relatively close between melanosomes and other areas). The average contrast-to-noise ratios (CNRs) for melanosomes are $49 \mathrm{~dB}$ and $25 \mathrm{~dB}$ in the PAM and OM images, respectively. The holes with few white dots inside the cells (PAM image) are nuclei, which is proved by staining them with 4',6diamidino-2-phenylindole and taking a fluorescence OM image [Fig. 2(a), right]. The nuclei are difficult to identify in the OM image due to the low contrast. Here the melanoma cells have irregular shapes because they were grown on glass. For typical red blood cells [Fig. 2(b)], the contrast disparity between PAM and OM can also be observed, although we could not find the same cells under the two microscopes. 
Then we imaged mouse ears in vivo with SW-PAM. All experimental animal procedures were carried out in conformity with the laboratory animal protocol approved by the Animal Studies Committee of Washington University in St. Louis. The melanin distribution in the ear (depilated) of a black mouse (Harlan Co., C57BL/6NHsd, 25 g, male) is shown in Fig. 3. Melanin synthesis occurs in melanosomes of melanocytes, and most melanocytes reside in the basal layer of the epidermis, whose thickness is about $10 \mu \mathrm{m}$ in this case [11]. Thus, we acquired two images focusing at $\sim 10 \mu \mathrm{m}$ and $\sim 30 \mu \mathrm{m}$ deep, respectively. In the shallower layer [Fig. 3 (a)], the single melanosomes can be clearly identified. In the deeper layer [Fig. 3(b)], most melanosomes are out of focus but more skin structures are shown, such as the sebaceous glands. Within this depth range we did not find an obvious decrease in resolution due to scattering. The signals from the deeper layer are weaker, but Fig. 3(b) appears to have higher contrast since it is scaled to the full color range. The OM image of the ear, although not shown here, has extremely low contrast. These results suggest potential applications of PAM in quantifying melanin distribution in vivo, which is important for detecting melanoma as well as determining individualized radiant exposure in dermatological laser therapies [12].

The ear of a nude mouse (Harlan Co., Hsd:Athymic Nude-Foxn1 ${ }^{\text {nu }}, 30 \mathrm{~g}$, male) was also imaged in vivo. Here, we used an objective with a $0.60 \mathrm{NA}$ (providing $400 \mathrm{~nm}$ resolution) instead because the thick layer of vessels required an extended focal zone. Since nude mice do not have much melanin in their skin, all the blood vessels, including capillaries, are shown with little background signals [Fig. 4(a)]. In some areas, we can see motionless red blood cells with the characteristic donut shape [Fig. 4(b)]. Because individual red blood cells can be imaged in vivo, SW-PAM can potentially be used to count red blood cells as an in vivo flow cytometer, measure blood flow velocity in capillaries, and monitor sickle cell disease. The same ear was imaged again, as shown in Fig. 4(c), 4 days after inoculation with $10 \mu \mathrm{l}$ of suspension containing 1 million B16 melanoma cells. The melanoma generated stronger photoacoustic signals than the vessels and was easily identified. The vasculature and melanoma have contrasts of $(12 \pm 1)$ : $1(33 \mathrm{~dB} C N R)$ and $(17 \pm 1): 1(36 \mathrm{~dB} \mathrm{CNR})$, respectively. If the laser wavelength is changed to, for example, $650 \mathrm{~nm}$, we can further increase the contrast difference between vasculature and melanoma. In the wide-field OM image [Fig. 4(d)], the melanoma is obscure, with a contrast of $(0.27 \pm 0.02): 1$ (21 dB CNR). Therefore, PAM has superior potential to detect melanoma in the early stage.

As shown by the results in Figs. 2-4, SW-PAM can resolve structures as small as subcellular organelles for both ex vivo and in vivo imaging. Additionally, by simply replacing the optical objective, our system can work with scalable imaging resolutions. Since the thickness of tissues that can be imaged is limited by the transmission-mode configuration, we are extending SWPAM to reflection mode for applications in more anatomical sites. As a result, SW-PAM along with the scaled-up macroscopy—deep-penetrating photoacoustic tomography [13,14]—may bridge microscopic research and clinical practice, especially for melanoma detection, vasculature visualization, reporter gene imaging [15], and sentinel lymph node mapping [16].

SW-PAM provides depth information based on the arrival time of photoacoustic signals. However, since we used a high-NA optical objective, the length of the optical focal zone is much smaller than that of the acoustic focal zone. To maintain high transverse resolution over a large depth range, we added $z$-scanning in the design [Fig. 1(a)]. With $z$-scanning, we can focus on different regions of interest, obtain two-dimensional images of those regions by selecting signals according to arrival times (see Fig. 3), and use the two-dimensional sections to form a three-dimensional view. Although the total scanning time will increase, high speed imaging can be achieved by replacing the mechanical scanning with laser scanning. These improvements may lead to more applications in the near future. 


\section{Acknowledgments}

We thank L. Li and Y. Wang for experimental assistance and helpful discussion, Y. Zhang for fixing and staining melanoma cells, D. Yao for help with optical microscopy, and M. Yang for preparing gold nanospheres. This work was sponsored in part by National Institutes of Health grants R01 EB000712, R01 EB008085, R01 CA113453901, U54 CA136398, and 5P60 DK02057933. L.W. has a financial interest in Microphotoacoustics, Inc. and Endra, Inc., which, however, did not support this work.

\section{References}

1. Wang LV. Multiscale photoacoustic microscopy and computed tomography. Nature Photon 2009;3:503-509.

2. Li C, Wang LV. Photoacoustic tomography and sensing in biomedicine. Phys. Med. Biol 2009;54:R59R97. [PubMed: 19724102]

3. Zhang HF, Maslov K, Stoica G, Wang LV. Functional photoacoustic microscopy for high-resolution and noninvasive in vivo imaging. Nature Biotechnol 2006;24:848-851. [PubMed: 16823374]

4. Maslov K, Zhang HF, Hu S, Wang LV. Optical-resolution photoacoustic microscopy for in vivo imaging of single capillaries. Opt. Lett 2008;33:929-931. [PubMed: 18451942]

5. Laufer J, Zhang E, Raivich G, Beard P. Three-dimensional noninvasive imaging of the vasculature in the mouse brain using a high resolution photoacoustic scanner. Appl. Opt 2009;48:299-306.

6. Kim C, Favazza C, Wang LV. In vivo photoacoustic tomography of chemicals: high-resolution functional and molecular optical imaging at new depths. Chem. Rev 2010;110:2756-2782. [PubMed: 20210338]

7. Wang, LV.; Wu, H. Biomedical Optics: Principles and Imaging. Wiley; Hoboken, NJ: 2007.

8. Wang LV. Tutorial on photoacoustic microscopy and computed tomography. IEEE J. Sel. Topics Quantum Electron 2008;14:171-179.

9. Gray-Schopher V, Wellbrock C, Marais R. Melanoma biology and new targeted therapy. Nature 2007;445:851-857. [PubMed: 17314971]

10. McDonald DM, Choyke PL. Imaging of angiogenesis: from microscope to clinic. Nature Med 2003;9:713-725. [PubMed: 12778170]

11. Kietzmann K, Lubach D, Heeren H-J. The mouse epidermis as a model in skin pharmacology: influence of age and sex on epidermal metabolic reactions and their circadian rhythms. Lab. Animal 1990;24:321-327.

12. Verkruysse W, Svaasand LO, Franco W. Remittance at a single wavelength of $390 \mathrm{~nm}$ to quantify epidermal melanin concentration. J. Biomed. Opt 2009;14:014005. [PubMed: 19256693]

13. Wang X, Pang Y, Ku G, Xie X, Stoica G, Wang LV. Noninvasive laser-induced photoacoustic tomography for structural and functional in vivo imaging of the brain. Nature Biotechnol 2003;21:803-806. [PubMed: 12808463]

14. Fronheiser MP, Ermilov SA, Brecht H, Conjusteau A, Su R, Mehta K, Oraevsky AA. Real-time optoacoustic monitoring and three-dimensional mapping of a human arm vasculature. J. Biomed. Opt 2010;15:021305. [PubMed: 20459227]

15. Li L, Zemp RJ, Lungu G, Stoica G, Wang LV. Photoacoustic imaging of lacZ gene expression in vivo. J. Biomed. Opt 2007;12:020504. [PubMed: 17477703]

16. Song KH, Stein EW, Margenthaler JA, Wang LV. Noninvasive photoacoustic identification of sentinel lymph nodes containing methylene blue in vivo in a rat model. J. Biomed. Opt 2008;13:054033. [PubMed: 19021413] 


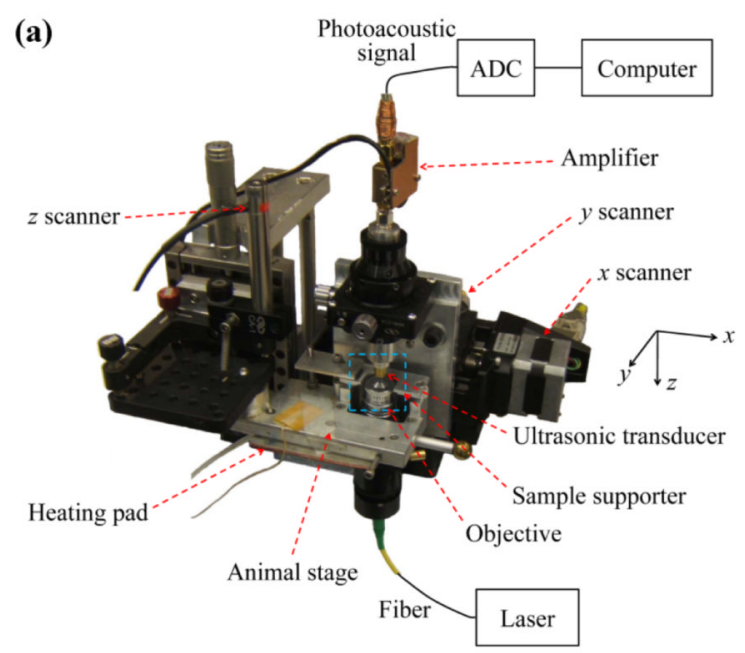

(b)

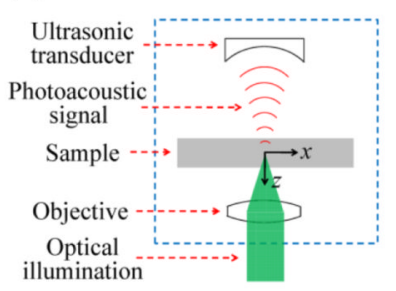

(c)

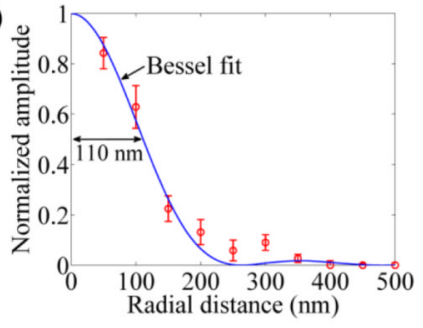

Fig. 1.

Subwavelength-resolution photoacoustic microscopy (SW-PAM). (a) Schematic diagram. (b) Close-up diagram showing the confocal structure of the optical objective and the ultrasonic transducer. (c) Point spread function of the system to measure the transverse spatial resolution. Red circle: the averaged pixel value. Blue line: the theoretical Bessel-form function. 
(a)

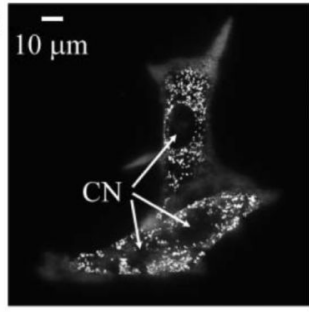

(b)

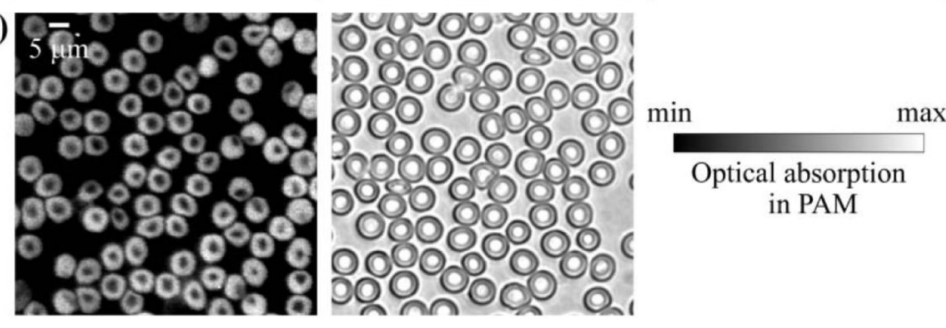

Fig. 2.

Ex vivo images of cells. (a) Melanoma cells grown on a cover glass and fixed. From left to right: photoacoustic microscopy (PAM) image, optical microscopy (OM) image (0.55 NA), and a composite of the PAM image and the fluorescence OM image of the stained nuclei (blue). In the PAM images the strong signals come mainly from melanin, and the white dots are melanosomes. CN: cell nucleus. (b) PAM and OM (1.0 NA) images of red blood cells. The strong signals in the PAM image come mainly from hemoglobin. 
(a)

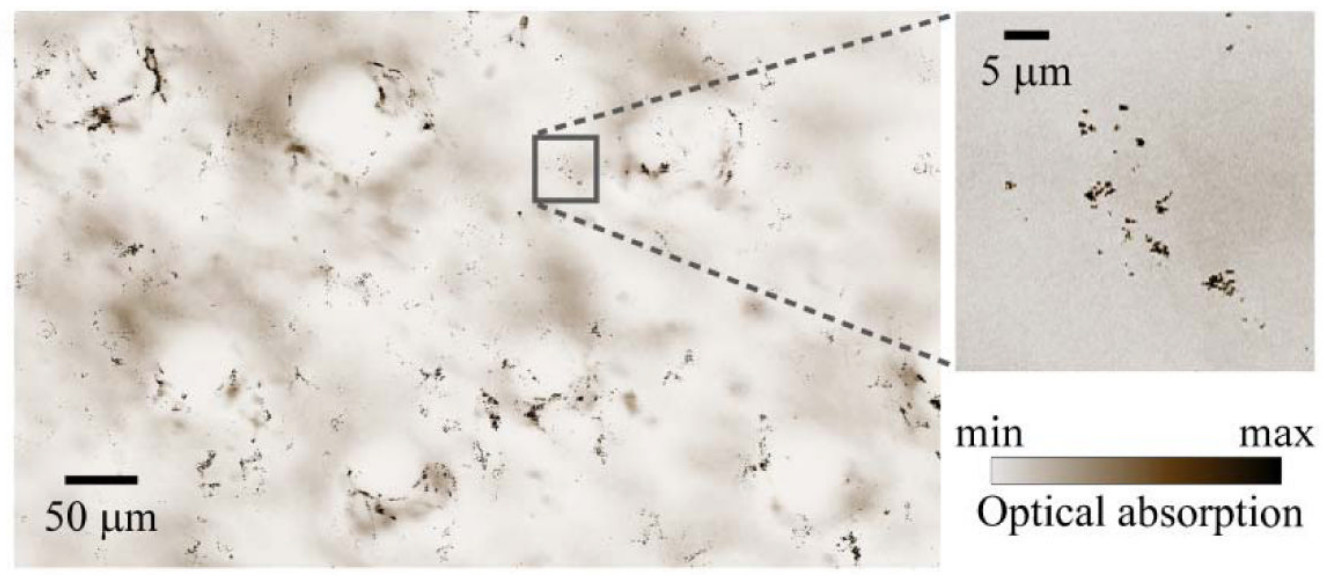

(b)

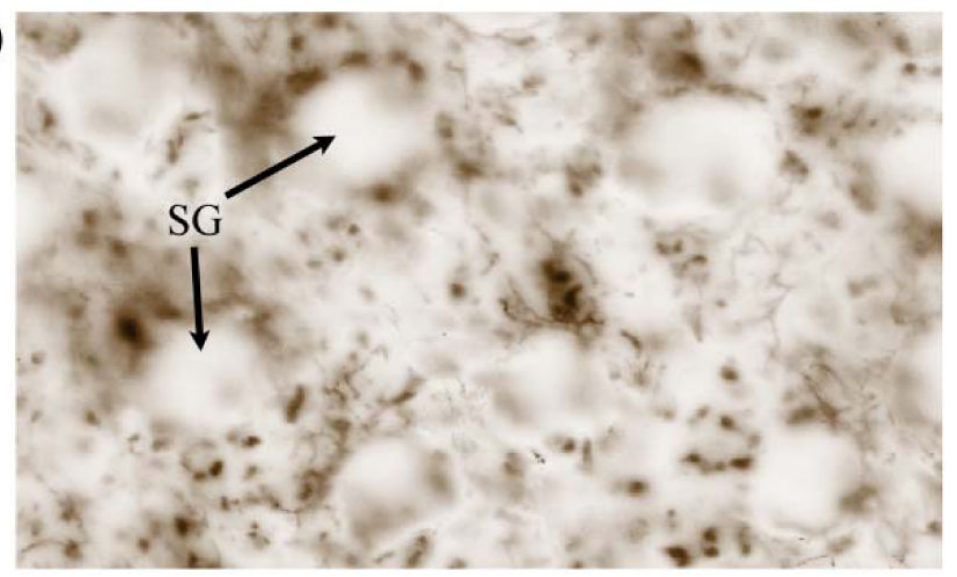

Fig. 3.

PAM images of a black mouse ear, showing the distribution of melanin. (a) Image focusing at $\sim 10 \mu \mathrm{m}$ deep (the close-up image indicates melanosomes). (b) Image focusing at $\sim 30 \mu \mathrm{m}$ deep. SG: sebaceous gland. 
(a)

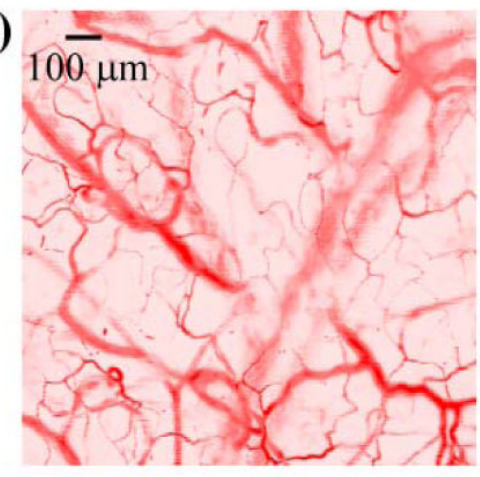

(c)

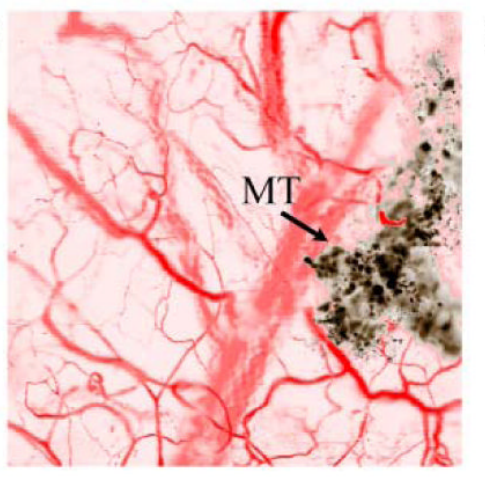

(b)

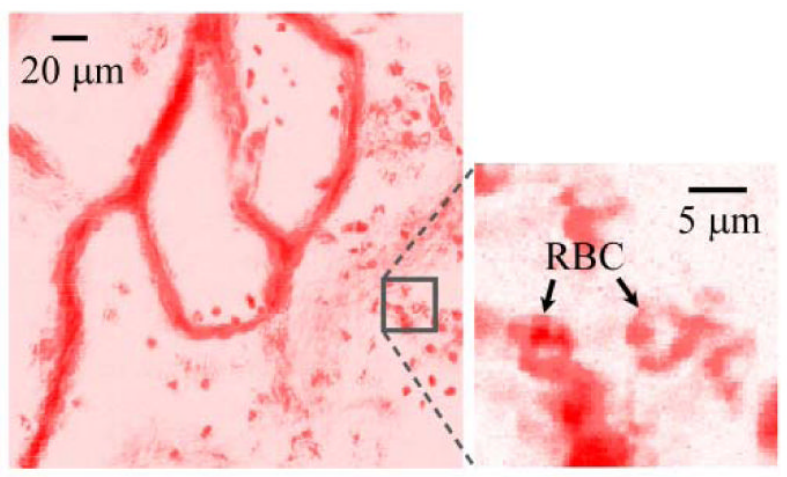

(d)
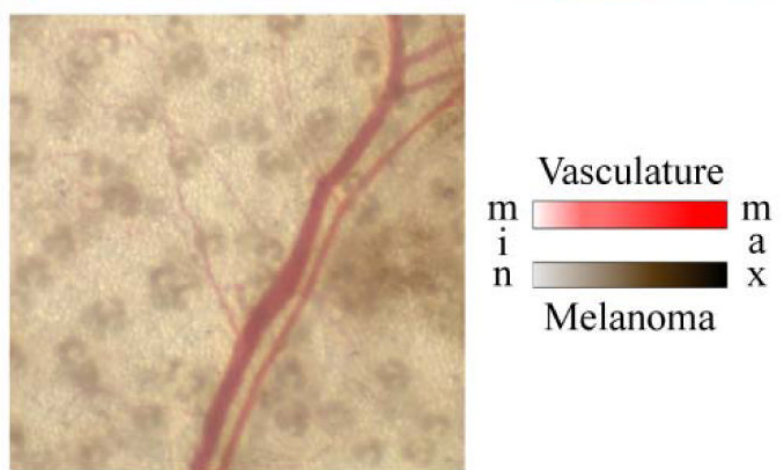

Fig. 4.

Monitoring of melanoma growing on a nude mouse ear. (a) PAM image of blood vessels in the ear acquired before injection of melanoma cells. (b) PAM image where in vivo red blood cells (RBCs) can be identified (the close-up image indicates the biconcave structure of RBCs). (c) PAM image of blood vessels and melanoma taken 4 days after injecting melanoma cells. The melanoma is extracted by taking the difference of the two PAM images [(a) and (c)] and is plotted in gray. MT: melanoma tumor. (d) OM image (0.057 NA) of the same area in (a) and (c). 